Service social

\title{
Théories du politique et pratique du service social
}

\section{Jean-Louis Gendron}

Volume 42, numéro 3, 1993

Perspectives théoriques

URI : https://id.erudit.org/iderudit/706629ar

DOI : https://doi.org/10.7202/706629ar

Aller au sommaire du numéro

Éditeur(s)

École de service social de l'Université Laval

ISSN

1708-1734 (numérique)

Découvrir la revue

Citer cet article

Gendron, J.-L. (1993). Théories du politique et pratique du service social. Service social, 42(3), 21-31. https://doi.org/10.7202/706629ar

\section{Résumé de l'article}

La question des liens théoriques qu'entretiennent le service social et l'analyse politique est très souvent posée, tant sous l'angle de la discipline que sous l'angle de l'intervention professionnelle. Se situant strictement d'un point de vue théorique et conceptuel, l'auteur aborde cette question, soulignant, à grands traits, l'évolution du service social dans la société québécoise. Partant de quelques conceptions différentes du politique, il montre d'abord comment le service social emprunte à l'une et à l'autre, pour ensuite déboucher sur la question, plus globale, des politiques sociales. 


\section{Théories du politique et pratique du service social}

Jean-Louis GENDRON

Professeur

École de service social, Université Laval

Le service social, qui est plus une pratique professionnelle qu'une discipline scientifique, puise une large part de ses connaissances aux théories des sciences humaines. Ainsi sont traditionnellement mis à contribution non seulement la psychologie et la sociologie, mais aussi le droit et même l'économique (Morin, 1958).

Mais qu'en est-il de la science politique? Quels rapports entretiennent entre elles les interventions du service social et les analyses politiques? C'est à ces questions que nous tenterons d'apporter ici quelques réponses, en précisant d'abord quel est l'objet fondamental autour duquel se construisent les théories du politique et comment celles-ci peuvent éclairer les pratiques du service social.

Pour ce faire, nous avons regroupé autour des thèmes suivants les idées émises dans le présent article: la structuration du pouvoir comme objet du politique; la régulation sociale comme objet du service social; et les politiques sociales comme jonction principale entre la structuration du pouvoir et les pratiques du service social. 


\section{LA STRUCTURATION DU POUVOIR COMME OBJET DU POLITIQUE}

La science politique, qui définit son objet comme étant l'étude des relations de pouvoir dans la société, nous offre du pouvoir toute une panoplie de définitions dont quelques-unes sont éminemment présentes derrière les pratiques du service social. En voici donc quelques-unes.

\section{Quelques conceptions différentes de la notion de pouvoir}

«Le pouvoir est la possibilité pour un acteur $A$ de faire poser à un acteur $B$ des gestes que ce dernier n'aurait pas posés sans l'influence de $A$ ” (Dahl, 1957). Depuis cette célèbre définition élaborée par Robert Dahl, en 1957, de nombreux auteurs ont contribué à faire de la structuration du pouvoir dans la société l'objet du politique, et de multiples théories se sont développées autour de cette idée. Dans son ouvrage Les cheminements de l'influence, Vincent Lemieux tente une synthèse de ces théories et les résume en huit types différents, allant des théories les moins spécifiquement politiques à celles qui situent le politique à tous les niveaux du processus organisationnel, lui donnant ainsi une dimension à la fois spécifique, englobante et holistique (Lemieux, 1979: 9).

Ainsi, par exemple, dans la typologie de Lemieux un auteur comme Maurice Duverger (1970) occuperait cette extrémité du continuum où le politique ne se distingue que très peu du droit et se limite à l'étude des institutions gouvernementales. Pour Duverger, en effet, à travers les institutions de l'État, l'étude des relations de pouvoir se limite à celles de l'appareil gouvernemental, et ce sont, finalement, les rapports institutionnels à caractère juridique, plus que les relations de pouvoir proprement dites, qui sont ici données comme objet de l'analyse politique. À l'autre extrémité, les théories d'un Michel Crozier (1977) donneraient du politique une conception tout aussi spécifique, quant à son objet, qu'universelle quant à son application empirique. Pour Crozier, les phénomènes de pouvoir sont essentiellement des phénomènes organisationnels où des acteurs sociaux négocient leur participation en utilisant au mieux les ressources qu'ils contrôlent pour atteindre leurs objectifs dans des structures où des règles normatives les encadrent plus ou moins partiellement. Utilisant, dans ce contexte, leur marge de liberté, les acteurs sociaux se livrent alors à des jeux de stratégies entre eux et avec l'organisation au sein de laquelle ils évoluent. Entre ces deux extrêmes, d'autres conceptions défendues par $d^{\prime}$ autres auteurs y auraient aussi leur place. Ainsi en est-il, d'après certains auteurs tout au moins, de la conception néo-marxiste du politique. Bien que celle-ci soit relativement étendue et universelle (et malgré l'apport de Paulantzas), le politique ainsi conçu n'est pas nécessairement spécifique 
parce qu'il emprunte trop exclusivement à une logique économique des rapports de production (Laborit, 1976).

La définition du politique, en effet, même en tenant pour acquis qu'elle a pour objet le phénomène du pouvoir dans la société, est traitée différemment par plusieurs écoles de pensée différentes. Ainsi, une perspective plus institutionnaliste définira le politique comme étant l'étude des phénomènes de pouvoir dans les grandes institutions politicojuridiques: le gouvernement, les ministères, les cours de justice, etc. En plus de Maurice Duverger dont nous avons évoqué les travaux, nous pourrions citer ici certains extraits de l'œuvre du politicologue québécois Gérard Bergeron, qui s'en rapproche parfois fortement (Bergeron, 1993 et 1965). De telles conceptions du politique et du pouvoir $n^{\prime}$ ont pas été absentes d'une certaine conception du service social québécois. À ses débuts, en effet, le service social tendait à se définir comme totalement apolitique. Seul le recours au gouvernement pour l'obtention discrétionnaire de subventions semblait ouvrir aux pratiques du service social la voie du politique (Perron, 1984).

\section{Pouvoir, politique et service social}

Une telle conception accommodait bien l'idéologie religieuse qui marqua le service social québécois de ses origines, jusqu'au milieu des années 60 . Le principe de la séparation de l'Église et de l'État était bien servi par une conception du politique qui limitait l'objet de celui-ci aux phénomènes étatiques et aux rapports organisationnels des grandes institutions gouvernementales. Ainsi, malgré le pouvoir qu'exerçait l'Église sur les valeurs et les pratiques du service social, nul n'aurait songé à soutenir que ce contrôle était de l'ordre du politique. Il s'agissait là de morale, d'éthique, mais non de politique. Le contrôle de l'Église était peu perçu comme politique et la notion de pouvoir était réservée à des phénomènes sociaux plus globaux, institutionnalisés, proches des appareils d'État et des processus gouvernementaux. La politique était d'ailleurs regardée avec dédain et il était peu tentant pour une profession naissante, comme le service social, de se définir dans une perspective politique. "La politique: cette "chose" que l'on n'osait nommer que du bout des lèvres» (Blouin, 1982)!

Une autre conception du politique, toutefois, se dégagera au milieu des années 60 et au début des années 70 pour secouer assez fortement cette conception institutionnaliste du pouvoir où le service social semblait s'être cantonné. La montée d'une analyse néo-marxiste de la lutte des classes conduisit plusieurs travailleurs sociaux, exerçant leurs fonctions en milieux populaires et communautaires, à introduire dans leurs analyses les approches du matérialisme historique. Fondée sur la théorie de la lutte des classes et sur des typologies québécoises de celle-ci, cette conception du 
politique se rapprochait beaucoup de l'économique par l'importance qu'elle accordait aux rapports de production. Dans cette perspective, en effet, les phénomènes de pouvoir étaient perçus comme la résultante du contrôle des moyens de production par une classe sociale dominante et oppressive. Cette optique, qui était largement celle de l'organisation communautaire, secouait fortement la perspective du casework qui dominait le service social de l'époque, et elle était fortement répudiée par les tenants de cette dernière approche. Dans les écoles et les départements de service social des universités, les débats entre les professeurs d'organisation communautaire et les autres, plus près des approches traditionnelles, se soldèrent quelquefois par des oppositions idéologiques quasi irréconciliables. D'un côté, l'engagement politique devenait inévitable; de l'autre, faire de la politique n'était pas le fait des travailleurs sociaux! Fomenter la révolution, encore moins!

Et pour cause! La notion de changement social qu'introduisait cette nouvelle façon de pratiquer le service social démolissait le socle traditionnel sur lequel avait reposé jusque-là une conception psycho-sociale du changement, qui s'accommodait assez bien d'une notion institutionnalisée du pouvoir. Même l'organisation communautaire s'était jusque-là trouvée fort à l'aise dans cette optique plutôt psycho-sociale. L'aménagement de ressources financées par des campagnes de souscription publiques constituait son principal secteur d'activité et il justifiait sa raison $d^{\prime}$ être par la nécessité de créer et de gérer des ressources à caractère d'aide psycho-sociale (Morin, 1962). L'histoire des conseils d'œuvres (ancêtres de Centraide) témoigne assez clairement de cela: "Le rôle d'un Conseil d'œuvres [...] (consistait) à faire prendre conscience à la communauté locale de l'importance du bien et du bonheur de chaque individu; à faire comprendre aux indigents, et aux œuvres mêmes et aux agences s'occupant du règlement des problèmes particuliers, que la solution de ces difficultés doit être essentiellement à base communautaire " (Giroux, 1957). Adaptation de l'individu à la société et création de ressources communautaires de bien-être.

L'inspiration plus néo-marxiste de la «nouvelle » organisation communautaire proposait de substituer à cela la voie d'un certain radicalisme où les problèmes sociaux seraient abordés sous l'angle des rapports de force entre groupes sociaux antagonistes. Elle introduisait ainsi une dimension politique différente dans les pratiques traditionnelles du service social, en posant que le politique n'était pas exclusivement phénomène gouvernemental institutionnel, mais phénomène de classes sociales et de groupes sociaux en opposition.

S'affrontaient donc ici deux conceptions radicalement opposées du politique: la conception institutionnaliste (Duverger), selon laquelle les 
rapports de pouvoir se limitent à des processus institutionnels gouvernementaux tellement officiels et légaux qu'ils laissent le service social indifférent dans ses idéaux professionnels, sauf peut-être en ce qui a trait à sa reconnaissance corporative; et une conception plus conflictuelle où les rapports de force découlant des luttes de classes ou de fractions de classes doivent être pris en compte par les travailleurs sociaux en tant qu'agents de changement.

Même si elle est demeurée à la marge des pratiques du service social, cette approche néo-marxiste a contribué plus que toute autre à poser la question des fondements politiques du service social et de la dichotomie expert / militant chez les travailleurs sociaux. Il devenait, en effet, impossible aux travailleurs sociaux de se positionner dorénavant comme « experts » de la pauvreté sans aborder la question parallèle de la " militance ». Et, très rapidement, il y eut débat quant au continuum expert professionnel / militant politique. L'expert devint davantage gestionnaire et le militant demeura organisateur communautaire. II faut dire, cependant, comme l'ont montré quelques articles de l'époque, que ce débat transgressa largement les cadres du service social pour s'étendre aussi à des disciplines comme la sociologie, la science politique et même la psychologie (Deslauriers, 1985 ; Desroches, 1981). II faut dire aussi que, dans la perspective des théories du politique que nous avons évoquées plus haut, le débat ne permettait pas toujours les nuances qui auraient permis de retrouver dans le modèle marxiste la spécificité du politique.

\section{LA RÉGULATION DES ACTEURS SOCIAUX ET LE POUVOIR DU SERVICE SOCIAL}

Pendant que s'affrontaient institutionnalistes et néo-marxistes, des arrangements politiques d'un autre ordre se préparaient à un autre niveau, et allaient proposer au service social une autre conception du jeu politique. Si le modèle d'analyse des acteurs sociaux les plus puissants $n^{\prime}$ est pas indifférent aux décisions qui seront imposées par la suite, la perspective systémique qui a prévalu au cours des années 70 , dans la définition par l'État des services sociaux, semble bien avoir été déterminante ici.

\section{Le pouvoir à un niveau microscopique}

Les recommandations de la commission Castonguay-Nepveu, les premières grandes grèves parapubliques et les resserrements idéologiques de la profession introduisaient le service social à une conception du politique tout à la fois plus étendue, plus englobante et pourtant plus spécifique que 
ne l'avaient été les précédentes. "Étendue » renvoie à une conception du politique qui reconnaît aux relations de pouvoir la caractéristique d'être présentes non seulement dans les appareils gouvernementaux, mais dans toutes les organisations humaines; "englobante" (ou holistique) signifie que ces relations de pouvoir maintiennent ensemble les éléments d'une organisation, en assurant sa gouverne ou son contrôle ; par "spécifique", on entend que ces relations ont une existence qui leur est propre et qui distingue le politique du social, de l'économique, du culturel, etc. (Lemieux, 1979: 4-10). En institutionnalisant les pratiques du service social, l'État en précisait inévitablement l'objet et forçait la profession à constater que cet objet $n^{\prime}$ était pas univoque, qu'il était au contraire porteur de plusieurs tendances et que celles-ci seraient défendues, à I'intérieur de la profession, avec acharnement par ceux et celles qui en étaient les adeptes. Ainsi, les luttes de pouvoir microscopiques, que l'on refusait jusque-là d'identifier à des phénomènes politiques, apparurent alors comme occupant presque tout l'espace de la discussion politique, ne laissant pratiquement aucune place pour l'engagement politique partisan ou extérieur au système de distribution des services sociaux lui-même. Les réformes de 1971, en effet, transférèrent le pouvoir aux technocrates de l'État et dépouillèrent largement tant les « experts " que les «militants » du service social. Apparut alors une troisième conception du politique, après celle des institutionnalistes et des néo-marxistes: I'approche stratégique. Cette troisième voie, c'est celle où le politique, conçu comme omniprésent au sein de tout rapport organisationnel, devient " étendu, spécifique et englobant» au sens où nous venons de le définir. La technocratisation du service social ramenait la discussion politique au niveau microscopique de I'action quotidienne (Renaud, 1978; Groulx, 1993).

\section{Le pouvoir: enjeu universalisé}

Dès lors, on rejoint ici les notions de pouvoir et de politique reliées aux phénomènes organisationnels, telles que certains auteurs les avaient déjà observées (Crozier, 1963). Selon cette conception du politique, l'acteur social, quel qu'il soit, ne pouvant s'abstenir ni de collaborer ni de $s^{\prime}$ imposer à l'intérieur du processus organisationnel où le plonge la lutte pour la survie, s'engage dans des structures de négociations où l'exercice du pouvoir devient un des éléments fondamentaux de son agir social (Crozier, 1977 ; Lemieux, 1989).

Dans une telle théorie du politique, les jeux du pouvoir sont l'ingrédient fondamental de toute structure d'action collective et il apparaît que les travailleurs sociaux $n^{\prime}$ ont pu y échapper, eux qui se retrouvaient de plus en plus à l'étroit dans des cadres de travail où leur profession, de 
quasi libérale (et quasi religieuse!) qu'elle avait été avant 1971, devenait de plus en plus technique et contrôlée. Soumise aux processus de régulation technocratique de l'État, scrutée à la loupe par toutes les commissions d'enquête, interpellée socialement et politiquement, la profession du service social semble s'être limitée à des jeux politiques microscopiques qui ne dépassèrent pas les seuils des sous-systèmes de distribution des services sociaux.

Dans un article paru en 1989, Gérald Doré et Lorraine Gaudreau se penchent sur la question et en tirent des conclusions intéressantes. Se posant la question de savoir si les travailleurs sociaux se confinent aux tâches qui leur sont assignées dans la prestation des politiques sociales ou bien s'ils interviennent dans le champ de l'action politique, ils écrivent ce qui suit:

Au Québec comme ailleurs, des intervenantes et intervenants sociaux (...) ont compris depuis longtemps [l'importance de déboucher sur la politique partisane], mais le manque de recherches à ce sujet contribue à y entretenir le préjugé [...] qu'il ne faut pas "mêler la politique avec le service social " (Doré et Gaudreau, 1989 : 258).

Il semble donc que, si les luttes de pouvoir - donc la politique - sont permises à un niveau microscopique, elles continuent d'être perçues comme tabou à un niveau socio-politique plus global. Le modèle de Crozier est acceptable; les modèles de Duverger et des néo-marxistes ne l'ont pas été. Les tenants de l'analyse systémique ont imposé non seulement leur pouvoir, mais leur conception du politique.

\section{LES POLITIQUES SOCIALES ET LA QUESTION DU POUVOIR EN SERVICE SOCIAL}

À la démarche qui précède et qui visait à saisir les aspects politiques du service social en le scrutant par l'intérieur de son idéal professionnel, il sera utile d'ajouter une deuxième perspective, celle des politiques sociales, qui consistera à le voir par l'extérieur. De fait, il est difficile et même impossible de séparer ces trois entités complémentaires d'un point de vue politique que sont les politiques sociales, les structures de pouvoir et la pratique du service social.

\section{Les politiques sociales: arène politique}

Comme nous l'avons vu, les travailleurs sociaux doivent de plus en plus considérer les politiques sociales comme encadrement systémique de 
leurs pratiques et, en ce sens, elles se différencient de moins en moins des autres politiques publiques, si ce n'est par l'objet de leur régulation qui est, finalement, la personne humaine elle-même. Quant au politique, comme nous l'avons vu aussi, il s'étend entre deux pôles, soit celui des processus gouvernementaux globaux et celui, plus microscopique, de la régulation organisationnelle.

Cette conception systémique des politiques sociales n'est pas étrangère à ce qu'auront été les idéaux de l'État-providence. Celui-ci, en effet, aura amené une nouvelle conception de ce qu'avaient été jusque-là non seulement les politiques sociales, mais I'ensemble des politiques publiques. Avec l'État-providence, ces dernières auront été conçues dans une perspective nettement systémique, $c^{\prime}$ est-à-dire comme des processus de régulation par lesquels l'État régit l'allocation des ressources dans la société. Les politiques sociales, en tant que secteur particulier des politiques publiques, ne différaient des autres que par l'objet de leur régulation, soit les personnes elles-mêmes, comme nous l'avons vu, en tant que ressources sociales particulières. Ainsi, par exemple, le cas typique des politiques de la main-d'œuvre en témoigne largement. (En signant actuellement ce que certains croient bien être l'arrêt de mort de cet État-providence, les gouvernements conservent comme objectif de créer d'importants programmes de formation de la main-d'œuvre. La régulation des personnes à travers certaines politiques publiques survivra donc à l'Étatprovidence s'il est vrai que celui-ci se meurt. On peut cependant se demander si le rapport du social à l'économique restera ce qu'il est maintenant.) C'est donc à cette catégorie toute spéciale de ressources sociales que sont les personnes que les politiques sociales se sont adressées, de même qu'aux ressources économiques jugées essentielles à leur bon fonctionnement social (Lemieux, 1989). Ainsi comprises, les politiques sociales auront délimité de façon de plus en plus linéaire la place du service social, l'impliquant principalement à la base du processus de régulation, $c^{\prime}$ est-à-dire à la phase de prestation des services. Dès lors, graduellement, la conception du pouvoir et du politique qu'adoptera la profession sera largement celle des effecteurs, c'est-à-dire de ces acteurs qui réalisent les pratiques décidées par $\mathrm{d}^{\prime}$ autres dans un système.

Cela conduira les décideurs de la profession à jouer leurs jeux de pouvoir dans des arènes qui sont celles de l'exécution et non des orientations idéologiques. Le processus de régulation auquel ils auront alors accès ne sera plus celui de la définition des grands objectifs sociaux, mais celui de l'exécution. II semble que dans I'histoire de la structuration du pouvoir qui a affecté l'évolution de la profession, l'influence technocratique que l'on perçoit à travers cette analyse systémique se soit plus imposée que celle, entre autres, du néo-marxisme. 
En définissant les politiques sociales comme régulatrices de l'ensemble de la société, d'autres preneurs de décisions, plus puissants dans l'Étatprovidence et la plupart du temps extérieurs à la profession, forcent ainsi les travailleurs sociaux à se positionner de cette façon. Ainsi, la prestation des politiques sociales devient de plus en plus I'unique champ d'action du service social, alors qu'à une époque pas si lointaine les travailleurs sociaux occupaient (ou rêvaient $d^{\prime}$ 'occuper) des domaines aussi variés que ceux des loisirs, du développement économique, de l'éducation, etc.

\section{Le pouvoir par régulation systémique}

Dans cette variance sectorielle d'autrefois, les travailleurs sociaux n'étaient pas soumis à la même régulation systémique de leurs postes et de leurs actions. Les organisations au sein desquelles ils se retrouvaient avaient souvent les caractéristiques de quasi-réseaux sociaux et les frontières n'étaient pas très étanches entre la décision, la consultation et l'exécution, de telle sorte que la profession conservait une vocation décisionnelle (Paré, 1988).

Dès lors qu'elle est plus systémique, la conception du politique qui se dégage chez les travailleurs sociaux devient plus précise et plus immédiatement intéressée. $C^{\prime}$ est la dimension stratégique du pouvoir qui devient alors l'élément moteur de la structuration politique. Les systèmes de régulation des politiques sociales où sont en effet insérés les travailleurs sociaux laissent vides de larges zones d'incertitude (Crozier et Friedberg, 1977) que les acteurs sociaux ont intérêt à occuper pour y préserver leurs acquis et y exercer leur influence sur les autres. Cette constatation sera facilement observable dans le réseau des affaires sociales au Québec et elle n'échappera pas aux investigations de la commission Rochon lorsque celle-ci parlera d'un système tenu en otage par l'intérieur. Engagé dans ce vaste système de régulation, le service social, comme les autres professions, a dû jouer le jeu des relations stratégiques de pouvoir, là où I'influence et les dimensions politiques de l'action collective ne peuvent être ignorées. Contrairement à d'autres professions, cependant, celle du service social ne semble pas avoir réussi à s'imposer dans des coalitions politiques puissantes qui lui auraient permis d'influencer pour la peine l'ensemble du processus politique, que ce soit au sein des partis, des groupes d'intérêt ou de la très haute administration publique.

Ainsi la dimension politique du travail social prend ici une place assez étendue et universelle, au sens où elle rejoint l'ensemble de cette profession, mais peu incisive dans l'ensemble du système politique. Cette dimension politique ne se joue pas aux niveaux officiels des institutions où même dans les partis politiques, donc en dehors des pratiques, mais 
à l'intérieur même de la tâche et du cadre de travail immédiat. La dimension politique du service social est donc strictement organisationnelle. Elle peut ainsi prendre des teintes différentes, allant de la collaboration pure et dure avec la technocratie locale à la concertation d'une DPJ, en passant par la rébellion discrète et le silence officiel, mais elle est assez silencieuse, en dehors des systèmes de distribution de services (Deslauriers, 1989; Langlois, 1993).

\section{CONCLUSION}

En conclusion, c'est donc une notion du politique pas plus institutionnaliste, mais beaucoup plus universelle et étendue que celle des débuts, qui inspire maintenant la pratique du service social. La relation de pouvoir, dans nos milieux de pratique comme ailleurs, est dorénavant considérée comme omniprésente dans l'ensemble du phénomène organisationnel qu'est l'intervention sociale. L'acteur social présent dans une organisation tente, en effet, $d^{\prime}$ 'utiliser au mieux les ressources qui sont les siennes, pour influencer les autres acteurs sociaux dans l'atteinte de ses propres objectifs: les travailleurs sociaux n'échappent pas à cette règle et cet exercice politique semble bien présent dans les milieux de pratique du service social.

Alors qu'avant l'avènement de l'État-providence la présence du politique, dans les systèmes de distribution des services sociaux, avait des allures de caméléons, se confondant fréquemment avec la diplomatie ecclésiastique, elle devient plus évidente sous la secousse néo-marxiste des années 70 . C'est ce thème que nous avons traité dans le premier volet de cet article. Puis, graduellement, I'apogée de l'État-providence lui imposera un caractère universellement systémique et microscopique, mais encore peu incisif dans la définition axiologique de la société. On peut se demander, par contre, si l'apprentissage du politique par les intervenants sociaux, comme toutes les autres formes d'intégration sociale dont les groupes sociaux comme les individus font l'expérience, ne les conduira pas maintenant à des variétés plus grandes d'engagements politiques.

Mais, s'étant trop souvent trompées, les analyses prospectives n'osent plus guère prédire l'avenir et cette conclusion suivra leur exemple. Les formes que prendront les engagements politiques futurs restent à découvrir. 


\section{Références bibliographiques}

BERGERON, Gérard (1965). Fonctionnement de l'État. Paris : Armand Colin.

BERGERON Gérard (1993). L'État en fonctionnement. Québec: PUL et l'Harmat$\tan$, p. 35-95.

BLOUIN, Jean (1982). Gérard Bergeron: de l'autre côté de l'action. Montréal: Nouvelle Optique, p. 46-47.

CROZIER, Michel (1963). Le phénomène bureaucratique. Paris: Seuil.

CROZIER, Michel et Erhard FRIEDBERG (1977). L'acteur et le système. Paris: Seuil.

DAHL, Robert A. (1957). "The Concept of Power ", dans Behavioral Science, p. 201-215.

DESLAURIERS, Jean-Pierre (1985). «De I'animation à la révolution », Service social, vol. 34, $n^{\circ} 2-3: 369-389$.

DESLAURIERS, Jean-Pierre (1989). "Honneur aux rebelles », Service social, vol. 38, $n^{\circ} 2-3: 263-270$.

DESROCHES, Henri (1981). "La recherche coopérative comme recherche-action », Actes du colloque sur la recherche-action, Chicoutimi, UQAC, octobre.

DORÉ, Gérald et Lorraine GAUDREAU (1989). "Politique sociale, politique partisane et profession ", Service social, vol. 38, $\mathrm{n}^{\circ}$ 2-3 : 246-262.

DUVERGER, Maurice (1970). Institutions politiques et droit constitutionnel. Paris : PUF.

GIROUX, Alphonse (1957). "Le Conseil des oeuvres et l'intégration de la profession du Service social dans la communauté », Service social, vol. 7, $\mathrm{n}^{\circ} 1$ : 3-17.

GroULX, Lionel-Henri (1993). Le travail social, analyse et évolution, débats et enjeux. Montréal: Éd. Agence d'Arc.

LABORIT, Henri (1976). Éloge de la fuite. Paris: Gallimard, p. 135-149.

LANGLOIS, Paul (1992). "Deux têtes et quelques doigts de pied: la réforme de la santé et des services sociaux", Nouvelles pratiques sociales, vol. $5, \mathrm{n}^{\circ} 1$ : 129-135.

LEMIEUX, Vincent (1979). Les cheminements de l'influence. Québec: PUL.

LEMIEUX, Vincent (1989). "Le pouvoir dans la réalisation des politiques sociales", Service social, vol. 38, $\mathrm{n}^{\circ}$ 2-3: 179-195.

MORIN, Claude (1958). "Économique et service social ou : Les étrangers dans la maison », Service Social, vol. 7, n $4: 155-164$.

MORIN, Claude (1962). "La méthode d'organisation communautaire dans le milieu canadien-français", Service social, vol. 11, n $1:$ 46-63.

PARÉ, Simone (1988). "L'École de service social de I'Université Laval, 19431988 ", dans Albert FAUCHER, Cinquante ans de sciences sociales à I'Université Laval. Sainte-Foy: Faculté des sciences sociales de l'Université Laval, p. 219-249.

PERRON, Jules (1984). "L'évolution des services sociaux d'expression française au Québec entre 1940 et 1960 ", Intervention, juillet, nº69:17-28.

RENAUD, Gilbert (1978). L'éclatement de la profession en service social. Montréal : Éd. coopératives Albert St-Martin, p. 125-145. 